\title{
Hepatic Microsomal Oxidative Metabolism of Pesticides and Other Xenobiotics in Pregnant $C D_{1}$ Mice
}

\author{
T. G. OSIMITZ ${ }^{1}$ AND A. P. KULKaRNI \\ Toxicology Research Laboratory, Department of Environmental and Industrial Health, School of Public \\ Health, The University of Michigan, Ann Arbor, Michigan 48109
}

Received April 18, 1984; accepted June 27, 1984

\begin{abstract}
Pregnancy-related changes in oxidative metabolism of several xenobiotics including pesticides were examined in the hepatic microsomes of $C D_{1}$ mice. The effect of pregnancy on hepatic microsomal cytochrome P-450-catalyzed substrate oxidation was found to be dependent upon the type of reaction examined. Not all substrates undergoing the same reaction showed identical changes during pregnancy. Those enzyme activities which exhibited a decline in specific activity during pregnancy generally exhibited no change in total hepatic capacity. Enzymes posting no change in specific activity throughout gestation generally showed large increases in total hepatic activity. Phorate $S$-oxidation was catalyzed by both microsomal flavin-containing monooxygenase (MFMO) and cytochrome $P$-450. Moreover, there was no pregnancy-related change in either MFMO or total enzymatic (MFMO plus cytochrome $P$-450) phorate $S$-oxidation. 1985 Academic Press, Inc.
\end{abstract}

\section{INTRODUCTION}

There have been several reports showing changes in the rates of hepatic microsomal cytochrome $P$-450-dependent xenobiotic oxidations during pregnancy $(1-10)$. These changes appear to be dependent upon species of animal, substrate examined, means of expressing data, and gestational age. All the available information originates from studies carried out with drugs or model compounds. Little is known about in vitro pesticide metabolism during pregnancy. Moreover, there are only two reports on the dynamics of the microsomal flavin-containing monooxygenase (MFMO) ${ }^{2}$ during pregnancy $(11,12)$. This pathway has been recently shown to be important in the sulfoxidation of thioether-containing organophosphorus insecticides (13). The relative

\footnotetext{
${ }^{1}$ Present address: Inhalation Toxicology Laboratory, Alberta Environmental Centre, Bag 4000, Vegreville, Alberta TOB 4LO, Canada.

${ }^{2}$ Abbreviations used: MFMO, microsomal flavincontaining monooxygenase; DMA, $N, N$-dimethylaniline; NOA, $n$-octylamine; EPN, $O$-ethyl- $O$-p-nitrophenylphenyl phosphonothioate
}

contribution of the microsomal cytochrome $P-450$ and MFMO in this reaction has not yet been documented.

In our earlier work (12) we reported the changes in the microsomal MFMO-dependent $\mathrm{N}$-oxidation of $\mathrm{N}, \mathrm{N}$-dimethylaniline (DMA) in various tissues from pregnant $\mathrm{CD}_{1}$ mice. In this paper, data obtained from hepatic microsomes for both the cytochrome $P$-450 and MFMO-mediated $S$-oxidation of the thioether moiety of the widely used pesticide, phorate, are presented. In addition, pregnancy-related changes in the hepatic microsomal cytochrome $P$-450-mediated oxidation of several xenobiotics, including pesticides, were investigated.

\section{MATERIALS AND METHODS}

Chemicals. Aldrin (recrystallized) was obtained from City Chemical (New York, N.Y.). The pesticides carbaryl, EPN ( $O$ ethyl-O-p-nitrophenyl phenylphosphonothioate), nicotine, parathion, and phorate were purchased from Chem Service (West Chester, Pa.), and were of the highest purity available. All other chemicals were of 
reagent grade and were obtained from Sigma Chemical Company (St. Louis, Mo.).

Animals. $\mathrm{CD}_{1}$ mice weighing $25-30 \mathrm{~g}$, purchased from Charles River Breeding Laboratories (Wilmington, Mass.), were used. Mice were maintained on a 12-hr day/ night cycle and had access to food and water ad libitum. The day a mating plug was observed was designated as Day 0 of pregnancy. Animals were sacrificed at Days 12 or 18 of pregnancy.

Tissue preparation. Immediately after sacrifice by cervical dislocation, livers were removed and homogenized in a medium containing ice-cold $0.25 M$ sucrose, $50 \mathrm{~m} M$ Tris buffer, $\mathrm{pH}$ 7.4, 0.1 mM EDTA. The homogenate was centrifuged at $9000 \mathrm{~g}$ for $20 \mathrm{~min}$, and the supernatant was decanted and centrifuged at $105,000 \mathrm{~g}$ for 60 min. The microsomal pellets were washed by resuspending in homogenization medium and recentrifuging at $105,000 \mathrm{~g}$ for 60 min. The washed microsomes were suspended in an appropriate buffer and used immediately for the different assays. Protein estimation was by the biuret method (14).

Enzyme assays. Published methods were used for assays of cytochrome $P-450$ (15); aldrin epoxidase (16); aminopyrine-, carbaryl-, and nicotine- $N$-demethylase (17); DMA-oxidase (12), EPN and parathion dearylase (18); and 7-ethoxycoumarin- $O$ deethylase (19). The optimum assay conditions for phoratc $S$-oxidation included incubation (total volume, $0.5 \mathrm{ml}$ ) of washed microsomes $(\sim 1.0 \mathrm{mg}$ protein $/ \mathrm{ml})$ in 100 $\mathrm{m} M$ Tris- $\mathrm{HCl}$ buffer, $\mathrm{pH} 7.4$, and a NADPH generating system consisting of $2.5 \mathrm{~m} M$ glucose 6-phosphate, $1.0 \mathrm{mM}$ $\mathrm{NADP}^{+}$, and 1 unit of glucose-6-phosphate dehydrogenase. Reaction mixtures were preincubated at $37^{\circ} \mathrm{C}$ with shaking for $3 \mathrm{~min}$ and reactions were started with the addition of phorate $(250 \mu M)$ in $2.5 \mu l$ acetone. Incubations were for 10 min with shaking at $37^{\circ} \mathrm{C}$. To discriminate between MFMO- and cytochrome $P$-450-mediated oxidations, 2.4 $\mathrm{m} M$ n-octylamine (NOA), an inhibitor of cytochrome $P-450$-catalyzed reactions (20), was added to some incubations. Reactions were terminated with $0.5 \mathrm{ml}$ ice-cold acetone. Phorate $S$-oxide was extracted with $2.5 \mathrm{ml}$ ethyl acetate (extraction efficiency, $>90 \%$ ). Quantification of phorate $S$-oxide was done by gas chromatography using a Varian Vista 4600 gas chromatograph, equipped with a 3-ft column of OV-101 and an electron capture detector. Gas chromatographic conditions were as follows: injector temperature, $220^{\circ} \mathrm{C}$, detector temperature, $300^{\circ} \mathrm{C}$ column oven temperature programmed from 150 to $190^{\circ}$ at $10^{\circ} / \mathrm{min}$; carrier gas (helium) flow, $30 \mathrm{ml} / \mathrm{min}$. Retention times for phorate, internal standard (methyl parathion), and phorate $S$-oxide were $2.7,4.3$, and $5.0 \mathrm{~min}$, respectively. Authentic phorate $S$-oxide (U.S. Environmental Protection Agency Reference Standard no. 5502) was used for calibration.

Data. Rates of xenobiotic metabolism are expressed relative to nonpregnant controls (represented on histograms as Day 0). The values reported are means $\pm \mathrm{SE}(n \geqslant$ 5 in all cases). Specific activity refers to nanomoles of product formed/minute/milligram microsomal protein. Total hepatic content represents nanomoles of product formed/minute/liver and was calculated by multiplying specific activity by microsomal yield per liver. Data was analyzed by a oneway analysis of variance (21). Comparison of means was done by the Bonferroni method (21). A $P$ value of $\leqslant 0.05$ was considered significant.

\section{RESULTS}

Compared to nonpregnant females, the body weight and the liver weight of pregnant mice increased by 34 and $47 \%$, respectively, by Day 12 of gestation (Table 1). The pregnant animals continued to gain weight; however, the percentage liver weight increase was minor on Day $18 \mathrm{com}$ pared to the total body weight. Although microsomal yield expressed on a milligram microsomes/gram liver basis did not change 
TABLE 1

Changes in Weight Values during Pregnancy

\begin{tabular}{lccc}
\hline & & \multicolumn{2}{c}{ Pregnant } \\
\cline { 3 - 4 } \multicolumn{1}{c}{ Parameter } & Nonpregnant & Day 12 & Day 18 \\
\hline Body weight $(\mathrm{g})$ & $28.4 \pm 0.5$ & $38.2 \pm 0.57^{*}$ & $50.9 \pm 1.8^{*}$ \\
& $(100)$ & $(134)$ & $(179)$ \\
Liver weight $(\mathrm{g})$ & $1.93 \pm 0.06$ & $2.83 \pm 0.10^{*}$ & $3.12 \pm 0.12^{*}$ \\
& $(100)$ & $(147)$ & $(162)$ \\
Microsomes $(\mathrm{mg}) /$ whole liver & $44.5 \pm 3.5$ & $61.7 \pm 2.8^{*}$ & $70.3 \pm 3.7^{*}$ \\
& $(100)$ & $(139)$ & $(158)$ \\
Microsomes $(\mathrm{mg}) / \mathrm{g}$ liver & $23.1 \pm 1.7$ & $21.8 \pm 0.5$ & $22.5 \pm 0.7$ \\
& $(100)$ & $(94)$ & $(97)$ \\
\hline
\end{tabular}

Note. Values represent means $\pm \mathrm{SE}(n=10)$. Relative values in parentheses below means.

* Significantly different from nonpregnant, $P<0.05$.

with gestation, total hepatic microsomal protein did rise in parallel with hepatic weight.

The specific content of cytochrome $P$ 450 remained unchanged throughout gestation (Fig. 1). However, the specific activity of aldrin epoxidase decreased by Day 18 to $57 \%$ of the level observed in nonpregnant mice. Total hepatic content of cytochrome $P-450$ increased with gestation $(+60 \%$ by Day 18 of gestation), whereas total hepatic aldrin epoxidase stayed constant.

Oxidative dearylation reactions were rather refractory to changes in the specific activity with only EPN-dearylase showing a significant $(30 \%)$ decline at Day 12 of gestation (Fig. 2). In contrast, the total capacity of the liver to dearylase EPN and parathion rose 57 and $69 \%$, respectively, over nonpregnant levels by Day 18 .

The specific activity of demethylases toward pesticide substrates declined significantly during pregnancy (Fig. 3). The specific activity of nicotine $\mathrm{N}$-demethylase decreased by $50 \%$ by Day 12 in contrast to a relatively modest $20 \%$ decrease in carbaryl $\mathrm{N}$-demethylase. As compared to the nonpregnant mice, both activities posted lower values at Day 18 of gestation. Despite the significant decreases observed in specific activities determined for both the substrates at Days 12 and 18 of gestation, the total hepatic capacity to perform oxidative demethylation was not altered except at Day 12 for nicotine.

The specific activity of aminopyrine $\mathrm{N}$ demethylase and 7-ethoxycoumarin- $O$-deethylase underwent comparable decreases $(-16$ and $-11 \%$, respectively) at Day 12 (Fig. 4). However, 7-ethoxycoumarin- $O$ deethylase activity declined considerably further by Day $18(-39 \%)$ than did ami-

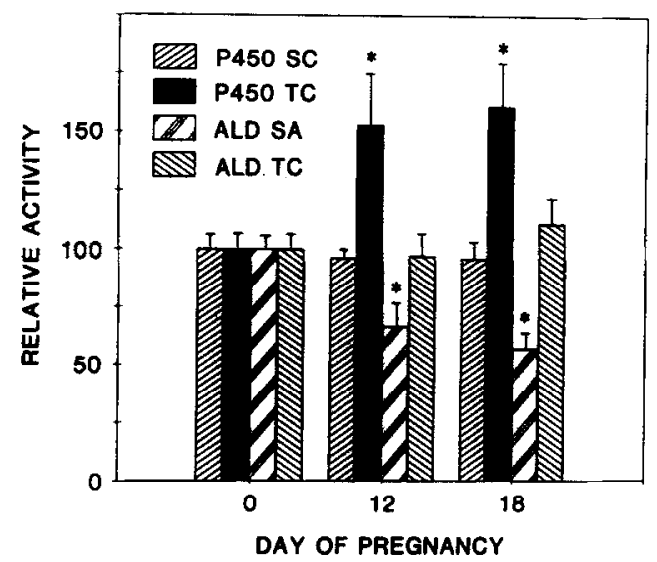

FIG. 1. Hepatic microsomal cytochrome P-450 and aldrin epoxidase during pregnancy. Values observed in nonpregnant mice were cytochrome $P-450$ specific content (P450 SC), $0.83 \pm 0.05$ nmollmg protein; total hepatic content of cytochrome P-450 (P450 TC), 37.4 \pm 2.2 nmol/liver; aldrin epoxidase specific activity (AE SA), $1.54 \pm 0.07 \mathrm{nmol} \mathrm{min}^{-1} \mathrm{mg}$ protein; total hepatic content of aldrin epoxidase, $63.8 \pm 4.0 \mathrm{nmol}$ min liver ${ }^{-1}$. Bars depict means $\pm S E(n \geqslant 5)$. *Significantly different from nonpregnant values; $P \leqslant 0.05$. 


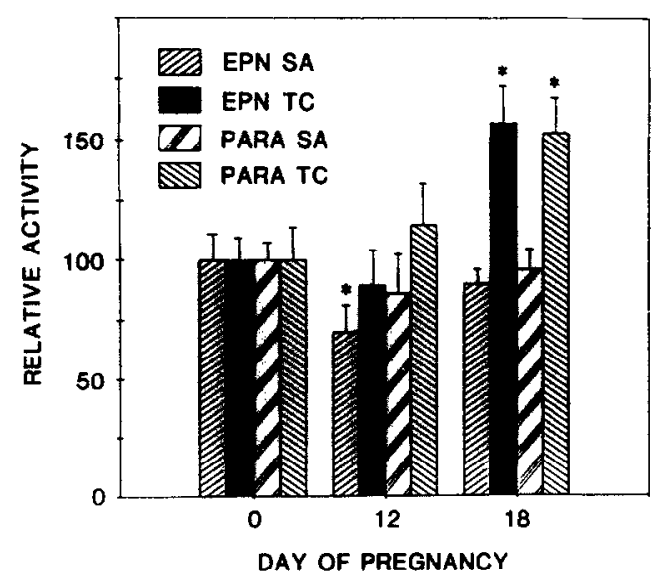

FIG. 2. Hepatic microsomal oxidative dearylation during pregnancy. Values observed in nonpregnant mice were EPN dearylase specific activity (EPA SA),

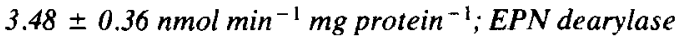
total hepatic content (EPN TC), $144 \pm 13.4 \mathrm{nmol}$ min $^{-1}$ liver ${ }^{-1}$; parathion dearylase specific activity (PARA SA), $2.41 \pm 0.19 \mathrm{nmol} \mathrm{min}^{-1} \mathrm{mg}$ protein $^{-1}$; parathion dearylase total hepatic content (PARA TC), $110 \pm 13.4 \mathrm{nmol} \mathrm{min}^{-1}$ liver $^{-1}$. Bars represent means $\pm S E(n \geqslant 5)$. *Significantly different from nonpregnant values; $P \leq 0.05$.

nopyrine $N$-demethylase ( $-20 \%$ ). Despite these changes in specific activities, there was no statistically significant change in the total hepatic capacity to dealkylate either substrate (Fig. 4).

Both hepatic MFMO and cytochrome $P$ 450 appear to catalyze the $S$-oxidation of phorate. If it is presumed that NOA causes complete inhibition of the cytochrome $P$ 450-mediated reaction, then it seems that a major portion (about $80 \%$ ) of the phorate sulfoxidation in hepatic microsomes from nonpregnant mice is catalyzed by the MFMO system (Fig. 5). However, the same concentration of NOA produced a $62 \%$ inhibition of aldrin epoxidase, a reaction known to be catalyzed by cytochrome $P$ 450 (22). The fact that NOA appears to cause only partial inhibition of cytochrome $P$-450-mediated metabolism suggests that a precise quantitation of the relative contribution of microsomal cytochrome $P-450$ and MFMO to phorate $S$-oxidation cannot be accomplished using NOA as the discriminating agent.
The data for the $S$-oxidation of phorate during pregnancy are given in Fig. 5. There was no change in the specific activity of the MFMO-catalyzed $S$-oxidation during gestation. In contrast, there was a statistically significant $(50 \%)$ decline in cytochrome $P$ 450-dependent activity by Day 12; however, activity increased to control levels by Day 18. The total hepatic content of MFMO catalyzed as well as total enzymatic (both the cytochrome $P-450$ plus MFMOdependent) phorate $S$-oxidation increased with gestation. The total hepatic cytochrome $P$-450-dependent activity, however, declined at Day 12 but recovered by Day 18.

\section{DISCUSSION}

Our observation that liver weight increases during pregnancy (Table 1 ) is consistent with that of other workers $(2-4,23)$. The elevation in liver mass is generally attributed to the proliferation of parenchymal cells (24) and, indeed, we have observed that this enlargement is accompanied by an increase in total microsomal yield per liver. This is important when evaluating xenobiotic metabolism during pregnancy since this increase may represent a concomitant increase in the xenobiotic-metabolizing enzyme complements of the tissue. However, in the past the question of whether the increase liver mass results in a parallel increment of xenobiotic-metabolizing enzymes has not been satisfactorily investigated.

The data presented show that there are several pregnancy-related changes in the hepatic microsomal oxidative enzymes in $\mathrm{CD}_{1}$ mice. These changes are substrate specific and depend upon how the activity is expressed, i.e, specific activity (nmol product $/ \mathrm{min} / \mathrm{mg}$ microsomal protein) or total hepatic activity (nmol product/min/ liver).

Although the specific content of cytochrome $P-450$ as determined by $\mathrm{CO}$ difference spectra does not change during pregnancy, we did note declines in the specific activity of the following reactions: $N$-deal- 


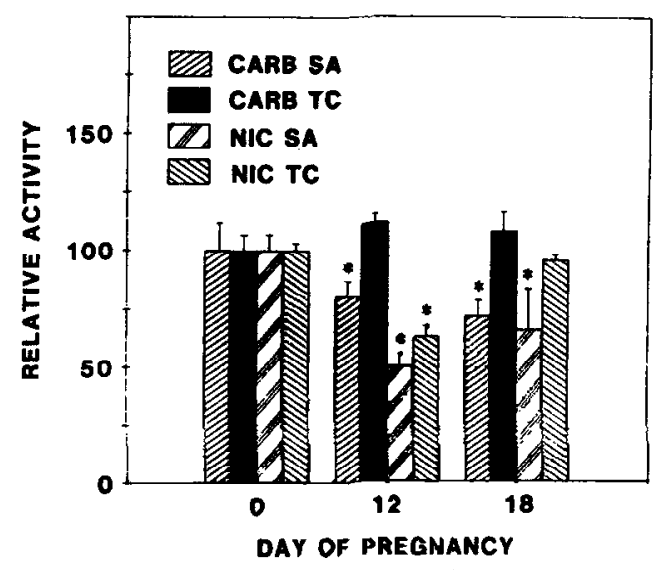

FIG. 3. Hepatic microsomal oxidative dealkylation during pregnancy. Values observed in nonpregnant mice were carbaryl demethylase specific activity (CARB SA), $0.89 \pm 0.12 \mathrm{nmol} \mathrm{min}^{-1} \mathrm{mg}$ protein $^{-1}$; total hepatic content of carbaryl demethylase (CARB TC), $35.4 \pm 6.2 \mathrm{nmol} \mathrm{min}^{-1}$ liver $^{-1}$; nicotine demethylase specific activity (NIC SA), $0.78 \pm 0.07 \mathrm{nmol}$ min $^{-1}$ mg protein $^{-1}$; total hepatic content of nicotine demethylase (NIC TC), $33.2 \pm 3.5 \mathrm{nmol} \mathrm{min}^{-1}$ liver ${ }^{-1}$. Bars represent means $\pm S E(n \geqslant 5)$. ${ }^{*}$ Significantly differeni from nonpregnant values, $P \leqslant 0.05$.

kylation of aminopyrine, carbaryl, and nicotine; epoxidation of aldrin; and $O$-dealkylation of 7-ethoxycoumarin. The finding that the specific content of cytochrome $P$ 450 does not change during pregnancy (Fig. 1) confirms previous reports $(3,5,9)$, but is in contrast to other investigations showing pregnancy-related decreases in the concentration of hepatic microsomal cytochrome P-450 $(1,2,4,6,8,23)$. A possibility exists that these conflicting results may be due to differences in the animal species, strains used, and the day of gestation on which the experiments were performed.

Significant differences were observed in the metabolism of substrates that undergo the same class of biotransformation during pregnancy (Fig. 2). Although the $N$-dealkylase activity toward aminopyrine, carbaryl, and nicotine decreased, the magnitude of decline was different with each substrate. These findings are consistent with the wellaccepted concept of cytochrome $P-450$ multiplicity in the hepatic microsomes (25), and suggest that distinct species of cytochrome $P-450$ may be involved in cata-

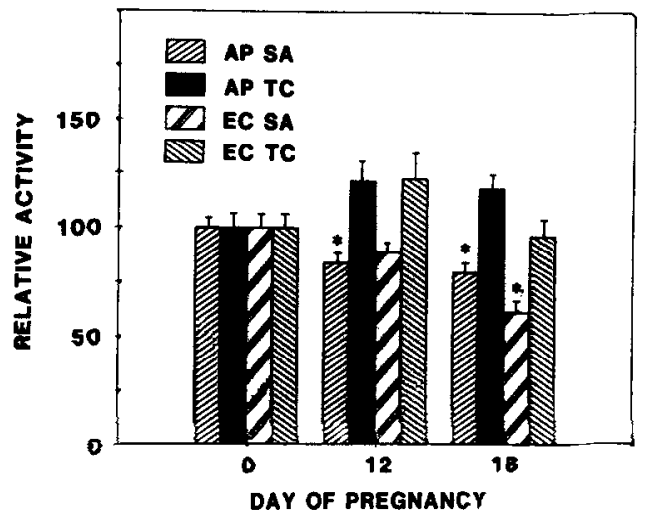

FIG. 4. Hepatic microsomal $N$-dealkylases during pregnancy. Values observed in nonpregnant mice were ethoxycoumarin deethylase specific activity $(E C$ $S A), 1.87 \pm 0.12 \mathrm{nmol} \mathrm{min}^{-1} \mathrm{mg}$ protein $^{-1}$; total hepatic content of 7-ethoxycoumarin-O-deethylase (EC TC), $85.5 \pm 4.7 \mathrm{nmol} \mathrm{min}^{-1} \mathrm{mg}_{\text {protein }}^{-1}$; aminopyrine demethylase specific activity $(A P S A), 6.81 \pm$ $0.28 \mathrm{nmol} \mathrm{min}^{-1} \mathrm{mg}$ protein ${ }^{-1}$; total hepatic content of aminopyrine demethylase $(A P T C), 345 \pm 22.9$ nmol $\min ^{-1}$ liver ${ }^{-1}$. Bars represent means $\pm S E(n \geqslant$ 6). *Significantly different from nonpregnant values; $P \leqslant 0.05$

lyzing the dealkylation of these xenobiotics. Pregnancy-related differences in the amount of enzyme present or in the modulation of the individual forms of cytochrome $P-450$ responsible for the demethylations may explain these results. Alternatively, there may be pregnancy-related changes in the rate-limiting steps in monooxygenation for one substrate and not another.

Unlike the specific activities for the substrates discussed above, there was no major pregnancy-related change in the specific activity of EPN or parathion dearylase (Fig. 2). Considered along with our earlier observation that the specific activity of hepatic microsomal cytochrome $P$-450-mediated DMA demethylation did not change during pregnancy (12), our present findings indicate that the depression of cytochrome $P-450$-mediated xenobiotic metabolism during pregnancy is not a general phenomenon but is specific for certain substrates.

The data showing no change in the specific activity of hepatic MFMO-dependent phorate $S$-oxidation during pregnancy (Fig. 


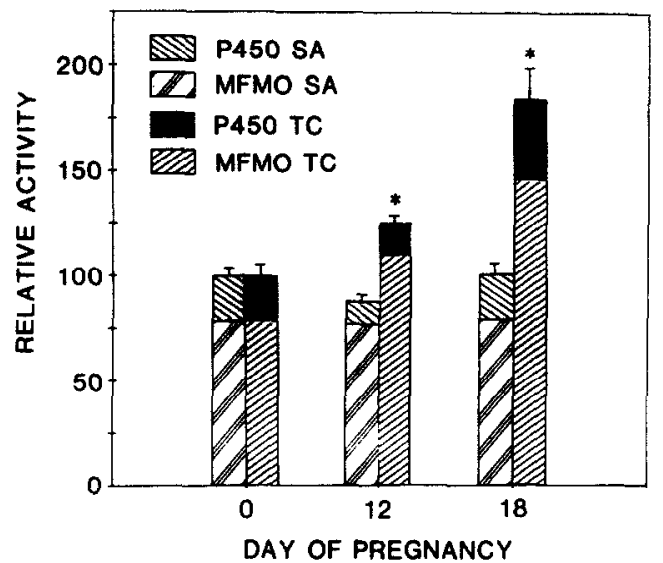

FIG. 5. Hepatic microsomal phorate $S$-oxidation during pregnancy. Values observed in nonpregnant mice were cytochrome $P-450$-dependent specific ac-

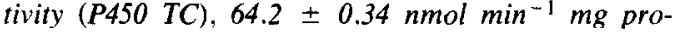
tein $^{-1}$; specific activity of MFMO-catalyzed phorate $S$-oxidase $(M F M O T C), 216 \pm 11.0 \mathrm{nmol} \mathrm{min}^{-1}$ liver ${ }^{-1}$. Bars represent means $\pm S E(n \geqslant 6)$. ${ }^{*}$ Significantly different from nonpregnant values; $P \leq 0.05$.

5) are consistent with our earlier report on the MFMO-catalyzed $\mathrm{N}$-oxidation of DMA (12). Taken together, these data imply that the MFMO may be insensitive to the endogenous modifiers present in pregnant animals that act to depress the rates of several cytochrome $P$-450-mediated xenobiotic oxidations.

In general, declines in the specific activity of several oxidative enzymes were offset by corresponding increases in liver weight during pregnancy. Hence, when considering the intact animal, it is possible that the overall oxidation of these substrates may not change with pregnancy. Indeed, Weitman and Lech (26) reported a $50 \%$ drop in the rate of hepatic elimination of parathion on a per gram liver basis; however, they did not find pregnancy-related differences in the total in situ elimination of parathion from perfused rat liver.

In contrast to the above situation, those substrates for which specific activity did not decline during pregnancy exhibited a substantial increase in the total hepatic capacity to oxidize the compounds. This may mean increased total detoxification for compounds such as EPN and parathion via oxidative dearylation in pregnant animals (27). Similar data are needed on the changes in the activities of other pathways involved in the metabolism of these pesticides and on changes in cholinesterase systems to better understand the overall toxicological significance of these observations. It is interesting to note that although we report no change in the specific activity of hepatic parathion dearylase during pregnancy, Weitman et al. (28) observed a $51 \%$ decline in the specific activity of hepatic microsomal parathion desulfuration during pregnancy in the mouse. The converse toxicological consequence is expected for a compound such as phorate which also exhibited a substantial increase in total hepatic $S$-oxidation during pregnancy. In this case, metabolism produces the phorate $S$ oxide, which is a more potent inhibitor of acetylcholinesterase than is the parent compound $(13,27)$.

\section{ACKNOWLEDGMENT}

This work was supported in part by Grant T32 ES07062 from the U.S. Public Health Service, National Institutes of Health.

\section{REFERENCES}

1. A. M. Guarino, T. E. Gram, D. H. Schroeder, J. B. Call, and J. R. Gillette, Alteration in kinetic constants for hepatic microsomal hydroxylase and ethylmorphine $\mathrm{N}$-demethylase associated with pregnancy in rats, J. Pharmacol. Exp. Ther. 168, 224 (1969).

2. M. G. Neale and D. V. Parke, Effects of pregnancy on the metabolism of drugs in the rat and rabbit, Biochem. Pharmacol. 22, 1451 (1973).

3. E. Schlede and R. Borowski, Decreased effect of phenobarbitol treatment on microsomal drug metabolizing enzyme activity during gestation, Nauyn-Schmiedeberg's Arch. Pharmacol. 281, 341 (1974).

4. M. E. Dean and B. H. Stock, Hepatic microsomal metabolism of drugs during pregnancy in the rat, Drug Metab. Dispos. 3, 325 (1975).

5. I. Gut, B. A. Becker, and M. Gutova, Effect of pregnancy on hepatic microsomal drug metabolism in rabbits and rats, Arch. Toxicol. 35, 41 (1976).

6. T. Tabei and W. L. Heinrichs, Hepatic steroid hydroxylase and aminopyrine $\mathrm{N}$-demethylase activities in pregnant rats and rabbits, and the ef- 
fect of phenobarbital, Biochem. Pharmacol. 25 , 2099 (1976).

7. G. Feuer, Action of pregnancy and various progesterones on hepatic microsomal activities, Drug Metab. Rev. 9, 147 (1979).

8. H. Mukhtar, R. M. Philpot, and J. R. Bend, Epoxide metabolizing enzyme activities and cytochrome P-450 content of rat ovaries during pregnancy, Biochem. Biophys. Res. Commun. 81, 89 (1978).

9. A. M. Symons, R. C. Turcan, and D. V. Parke, Hepatic Drug Metabolism in the Pregnant Rat, Xenobiotica 12, 365 (1982).

10. R. G. Turcan, P. P. Tamburini, G. G. Gibson, D. V. Parke, and A. M. Symons, Drug metabolism, cytochrome $\mathrm{P}-450$ spin state, and phospholipid changes during pregnancy in the rat, Biochem. Pharmacol. 30, 1223 (1981).

11. T. R. Devereux and J. R. Fouts, Effect of pregnancy or treatment with certain steroids on $\mathrm{N}, \mathrm{N}$-dimethylaniline demethylation and $\mathrm{N}$-oxidation by rabbit liver or lung microsomes, Drug. Metab. Dispos. 3, 254 (1975).

12. T. G. Osimitz and A. P. Kulkarni, Oxidative metabolism of xenobiotics during pregnancy: significance of microsomal flavin-containing monooxygenase, Biochem. Biophys. Res. Commun. 109, 1164 (1982).

13. N. P. Hajjar and E. Hodgson, Sulphoxidation of thioether-containing pesticides by the flavincontaining monooxygenase of pig liver microsomes, Biochem. Pharmacol. 31, 745 (1982).

14. A. G. Gornall, C. S. Bardwill, and M. M. David, Determination of serum proteins by means of the biuret reaction, J. Biol. Chem. 117, 751 (1949).

15. T, Omura and R. Sato, The carbon monoxide binding pigment of liver microsomes, J. Biol. Chem. 239, 2370 (1964).

16. T. Wolff, D. Erhard, and H. Wanders, Aldrin epoxidation, a highly sensitive indicator specific for cytochrome P-450-dependent monooxygenase activities, Drug Metab. Dispos. 7, 301 (1979)

17. A. P. Kulkarni and E. Hodgson, Mouse liver microsomal hexose-6-phosphate dehydrogenase:
NADPH generation and utilization in monooxygenation reactions, Biochem. Pharmacol. 31, 1137 (1982).

18. A. A. Nomeir and W. C. Dauterman, In Vitro metabolism of EPN and EPNO by mouse liver, Pestic. Biochem. Physiol. 10, 190 (1979).

19. A. Aitio, A simple and sensitive assay of 7-ethoxycoumarin deethylation, Anal. Biochem. 85, 488 (1978).

20. C. Jefcoate, J. L. Gaylor, and R. L. Calabrese, Ligand interactions with cytochrome P-450. Binding of primary amines, Biochemistry 8 , 3455 (1969).

21. J. Neter and W. Wasserman, "Applied Linear Statistical Models," p. 419, Richard D. Irwin, Homewood, Illinois, 1974.

22. T. Wolff, H. Greim, M. Huang, G. T. Miwa, and A. Y. H. Lu, Aldrin epoxidation catalyzed by purified rat-liver cytochromes P-450 and P-448, Eur. J. Biochem. 111, 545 (1980).

23. M. J. Vodicnik, C. R. Elcombe, and J. J. Lech, The transfer of $2,4,5,2^{\prime}, 4^{\prime}, 5^{\prime}$-hexachlorobiphenyl to fetuses and nursing offspring. II. Induction of hepatic microsomal monooxygenase activity in pregnant and lactating mice and their young, Toxicol. Appl. Pharmacol. 54, 301 (1980).

24. R. M. Campbell, B. F. Fell, and W. S. Mackie, Ornithine decarboxylase activity, nucleic acids, and cell turnover in the livers of pregnant rats, J. Physiol. (London) 241, 699 (1974).

25. M. J. Coon and A. V. Persson, Microsomal cytochrome P-450: a central catalyst in detoxication reactions, in "Enzymatic Basis Detoxication" (W. J. Jakoby, Ed.) Vol 1, p. 117, Academic Press, New York, 1980.

26. S. Weitman and J. Lech, Influence of pregnancy on the hepatic metabolism of parathion, Toxicologist 3, 78 (1983).

27. M. Eto, "Organophosphorus Pesticides: Organic and Biological Chemistry,' CRC Press, Cleveland, 1974.

28. S. D. Weitman, M. J. Vodicnik, and J. J. Lech, Role of pregnancy on the activation and detoxification of parathion, The Pharmacologist 24, 93 (1982). 